\title{
USING ULTRA LONG PERIOD CEPHEIDS TO EXTEND THE COSMIC DISTANCE LADDER TO 100 Mpc AND BEYOND
}

\author{
Jonathan C. Bird, K. Z. Stanek, and José L. Prieto \\ Department of Astronomy, The Ohio State University, 140 West 18th Avenue, Columbus, OH 43210, USA; bird@astronomy.ohio-state.edu, \\ kstanek@astronomy.ohio-state.edu,prieto@astronomy.ohio-state.edu \\ Received 2008 August 1; accepted 2009 January 14; published 2009 April 3
}

\begin{abstract}
We examine the properties of 18 long period (80-210 days) and very luminous (median absolute magnitude of $M_{I}=-7.86$ and $\left.M_{V}=-6.97\right)$ Cepheids to see if they can serve as a useful distance indicator. We find that these Ultra Long Period (ULP) Cepheids have a relatively shallow period-luminosity (PL) relation, so in fact they are more "standard candle" like than classical Cepheids. In the reddening-free Wesenheit index, the slope of the ULP PL relation is consistent with zero. The scatter of our sample about the $W_{I}$ PL relation is 0.23 mag, approaching that of classical Cepheids and Type Ia Supernovae. We expect this scatter to decrease as bigger and more uniform samples of ULP Cepheids are obtained. We also measure a nonzero period derivative for one ULP Cepheid (SMC HV829) and use the result to probe evolutionary models and mass loss of massive stars. ULP Cepheids' main advantage over classical Cepheids is that they are more luminous, and as such show great potential as stellar distance indicators to galaxies up to $100 \mathrm{Mpc}$ and beyond.
\end{abstract}

Key words: Cepheids - distance scale - stars: distances - stars: mass loss

Online-only material: color figures

\section{INTRODUCTION}

A reliable method of measuring the physical distance to astrophysical objects has always been sought after in observational astronomy (e.g., Bessel 1839). In the era of "precision cosmology," the need for accurate physical distance measurements has been amplified (e.g., Spergel et al. 2003; Riess et al. 2004; Tegmark et al. 2004). Accurate and precise distance indicators hold the key to pinning down the value of the Hubble constant $\left(H_{0}\right)$ and many other cosmological parameters (see discussion in, e.g., Macri et al. 2006). A number of methods have been employed to determine extragalactic distances, with varying degree of success (e.g., Freedman et al. 2001). The construction and reliability of the "cosmological distance ladder" depends crucially on Cepheid variables being able to provide precise and accurate distances to nearby ( $d \lesssim 20 \mathrm{Mpc}$ ) galaxies. The quest for such distances has been an arduous journey for almost a hundred years, with many dramatic twists and turns (for a review of early years, see Baade 1956; for a recent review see e.g., Macri 2005).

Cepheids offer several advantages as distance indicators. Massive stars $\left(\geqslant 5 M_{\odot}\right)$ make an excursion through the instability strip and most, if not all, of them become Cepheid variables. These variable stars are relatively bright $\left(M_{V} \sim-4\right.$ for a $P \sim 10$ day Cepheid) and often have large brightness variations (amplitude $\sim 1 \mathrm{mag}$ ) with a characteristic "saw-tooth" shaped light curve. Their intrinsic brightness, combined with their light-curve shape and colors, make them easy to distinguish from other classes of variable stars. As a result, Cepheids have been detected and studied in a significant number of star-forming galaxies. The physical mechanisms underlying Cepheid pulsation are well understood, including the observed tight period-luminosity (PL) relationship (e.g., Chiosi et al. 1993). The small scatter in the PL relation allows distance measurements precise to 5\% (e.g., Macri et al. 2006). For these reasons, Cepheids are commonly used to calibrate other distance indicators, forming the base of the cosmological distance ladder.
Despite their many advantages as a distance indicator, Cepheid distances also have some shortcomings. Most Cepheids have an intrinsic brightness of $M_{V} \geqslant-5$, so with the current instrumentation they can be only used to measure distances to $\lesssim 30 \mathrm{Mpc}$ (the largest Cepheid distance in Freedman et al. 2001 is $\sim 22 \mathrm{Mpc}$ ). Observations of Cepheids in distant galaxies are also hindered by blending (Mochejska et al. 2000) —as young stars, Cepheids live in close proximity to the crowded star-forming regions of their host galaxies, and are thus likely to have another star of similar brightness on the scale of a typical instrumental point-spread function (PSF). The effect of blending becomes worse as the square of the distance to the host galaxy (Stanek \& Udalski 1999), again limiting the usefulness of Cepheids to measuring distances $\lesssim 30 \mathrm{Mpc}$ even with high-resolution instruments such as the Hubble Space Telescope (HST). Ideally, we would like to find a distance indicator that shares the good properties of classical Cepheids, but is even more luminous, allowing us to observe it further away and be less susceptible to blending. In this paper, we discuss such a possible distance indicator, namely Ultra Long Period (ULP) Cepheids.

We define ULP Cepheids as fundamental mode Cepheids with pulsation periods greater than 80 days. Several such Cepheids have been already discovered in the pioneering study of Leavitt (1908). However, ULP Cepheids have traditionally been ignored for distance measurements as they are PL outliers. Indeed, the observed PL relation flattens for Cepheids with periods greater than 100 days (e.g., Grieve et al. 1985; Freedman et al. 1992). Grieve et al. (1985) suggest that long period Leavitt Variables could be used for distance measures - unfortunately this idea has not permeated through the community. We argue that the flattening of the PL at long periods actually improves the usefulness of ULP Cepheids as distance indicators because it makes them a good standard candle in the traditional sense. We note several additional advantages of ULP Cepheids over lower period Cepheids due to their increased luminosity. ULP Cepheids could be used as a stellar distance measure to the Hubble flow (up 
to $\sim 150 \mathrm{Mpc}$ ) — several times the current observational limit. In Section 2, we describe our sample compiled from the literature. The ULP Cepheid PL relation is discussed in Section 3. Section 4 demonstrates how ULP Cepheids may provide the additional benefit of testing massive stellar evolutionary models. We summarize our results in Section 5.

\section{SAMPLE}

We have assembled a sample of ULP Cepheids from the literature and list their reported positions, periods, and mean $V$ and $I$ magnitudes (see Table 1). We adopt the periods, reddening values, and distance moduli found in these sources except in the case of the Magellanic Clouds (see below). Our primary criteria for selecting the sample was the existence of $V$ and $I$ data calibrated on the standard Johnson/Kron-Cousins magnitude system using Landolt standards (with the possible exception of the Magellanic Clouds; see below). The ULP distinction is applied to fundamental mode Cepheids with periods greater than 80 days. We combed the recent literature for reports of such variable stars.

Magellanic Clouds. Our sample includes four Large Magellanic Cloud (LMC) and three Small Magellanic Cloud (SMC) ULP Cepheids. Freedman et al. (1985) calibrated photoelectric observations of these Cepheids and transformed them to the Johnson/Kron-Cousins standard system. The mean fluxweighted photometry for the six Cepheids reported in Freedman et al. (1985) agrees with the Landolt standard star calibrated results of Moffett et al. (1998) to within 0.04 mag, suggesting that the standard photometric system calibration is robust. Mean flux-weighted photometry for HV1956 is obtained from the All Sky Automated Survey (ASAS; Pojmanski 1997) and Moffett et al. (1998). The $V$ light curves of six of these ULP Cepheids were obtained from ASAS. HV2883 was not targeted by ASAS, and its $V$ light-curve photometry was obtained from Madore (1975), van Genderen (1983), and Moffett et al. (1998). Moffett et al. (1998) provided the $I$ light curve data for the entire sample. We applied the analysis of variance technique (SchwarzenbergCzerny 1989) to the seven Harvard Variable light curves in Figure 1 to obtain the periods listed in Table 1. We adopt total reddening values of $E(B-V)=0.14$ mag and $E(B-V)=0.09$ mag for the LMC and SMC, respectively (Udalski et al. 1999). We assume a distance modulus (DM) of $(m-M)_{0}=18.5 \mathrm{mag}$ to the LMC for consistency with the sources listed in Table 1. The SMC DM used is $(m-M)_{0}=18.93 \mathrm{mag}$ (Hilditch et al. 2005; Keller \& Wood 2006). The LMC (SMC) hosts ULP Cepheids with periods of 98.6, 109.2, 118.7, and 133.6 (84.4, 127.5 , and 210.4) days. The LMC has gas-phase oxygen abundance $12+\log (\mathrm{O} / \mathrm{H})=8.39 \pm 0.10$ (Pagel et al. 1978), while the $\mathrm{SMC}$ is $12+\log (\mathrm{O} / \mathrm{H})=7.98 \pm 0.10$ (Peimbert \& TorresPeimbert 1976).

The Araucaria Project (Pietrzyński et al. 2002) is a photometric survey of Local and Sculptor Group galaxies and their Cepheid populations. The primary goal is to more accurately determine the distances to these galaxies and to characterize the dependence of various stellar distance indicators on metallicity and age. The Araucaria Project has observed ULP Cepheids in the following galaxies.

NGC 55. Five ULP Cepheids were found in NGC 55 (Pietrzyński et al. 2006). Observations were taken with the Optical Gravitation Lensing Experiment (OGLE) detector on the Warsaw $1.3 \mathrm{~m}$ telescope at Las Campanas Observatory. They estimate that the calibration procedure used to transform their photometric data from the OGLE filters to the standard system pro- duced errors $\leqslant 0.03 \mathrm{mag}$. Follow-up observations in the infrared (IR) revealed a total reddening of $E(B-V)=0.13 \mathrm{mag}$ (Gieren et al. 2008). Their multiwavelength PL analysis produced a DM to NGC 55 of $26.43 \pm 0.04 \pm 0.08$ mag (statistical and systematic errors, respectively). NGC 55 hosts ULP Cepheids with periods of $85.1,97.7,112.7,152.1$, and 175.9 days. The oxygen abundance of NGC 55 is $12+\log (\mathrm{O} / \mathrm{H})=8.05 \pm 0.10$ (Tüllmann et al. 2003).

NGC 6822. One ULP Cepheid was found in NGC 6822 (Pietrzyński et al. 2004). The filters and telescope used are identical to those of NGC 55 (Pietrzyński et al. 2006). Similarly, the reported calibration error onto the standard system is $\leqslant 0.03$ mag. As in the multiwavelength follow-up study of NGC 6822 (Gieren et al. 2006), we adopt a total reddening of $E(B-V)=0.36 \mathrm{mag}$. The lone ULP Cepheid in NGC 6822 has a period of 123.9 days. Gieren et al. (2006) calculate a DM to NGC 6822 of $23.31 \pm 0.02 \pm 0.06$ mag (statistical and systematic errors, respectively). NGC 6822 has a similar oxygen abundance to NGC 55 of $12+\log (\mathrm{O} / \mathrm{H})=8.11 \pm 0.10$ (Peimbert et al. 2005).

$N G C$ 300. Gieren et al. (2004) found three ULP Cepheids in NGC 300. Again, OGLE filters were used for the observations. Their calibration onto the standard system has a reported error $\leqslant 0.03$ mag. A multiwavelength study of NGC 300 (Gieren et al. 2005 ) determined a reddening-free $\mathrm{DM}$ of $26.37 \pm 0.05 \pm 0.03$ mag (statistical and systematic, respectively) using a total reddening of $E(B-V)=0.10$ mag. ULP Cepheids of 83.0, 89.1, and 115.8 days are observed in NGC 300. NGC 300 has a strong metallicity gradient; therefore, we adopt a mean Cepheid radial distance of $4 \mathrm{kpc}$ and apply the averaged gradient of Urbaneja et al. (2005) to obtain a mean oxygen abundance value of $12+\log (\mathrm{O} / \mathrm{H})=8.25 \pm 0.22$.

I $Z w$ 18. Aloisi et al. (2007) discovered three ULP Cepheids from the extremely metal-poor galaxy I $\mathrm{Zw} 18$, though they could not confirm one candidate. A follow-up study (Fiorentino et al. 2008) presents flux-weighted mean photometry but no data, so the light curves of these objects could not be included in Figure 1. The ULP Cepheids have periods of 129.8 and 125.0 days (Table 1). After accounting for extinction $E(B-$ $V)=0.032$ mag, Aloisi et al. (2007) used the red giant branch (RGB) tip to determine a DM of $31.30 \pm 0.17 \mathrm{mag}$, while Fiorentino et al. (2008) found a DM of $31.35 \pm 0.26 \mathrm{mag}$ via pulsation models. We use the former measurement as it is considered more reliable by the authors. We do not include these two Cepheids in the upcoming PL determination as I Zw 18 is a full dex more metal-poor than the other galaxies in this sample $(12+\log (\mathrm{O} / \mathrm{H})=7.2 \pm 0.10 ;$ Skillman \& Kennicutt 1993). There is an increasing amount of support for a metallicity dependent PL (e.g., Sandage et al. 2008) and including these Cepheids in our PL analysis would greatly increase the metallicity dispersion of the host galaxies in our sample. We do, however, make use of them to examine the ULP PL relation dependence on metallicity.

\subsection{Absolute Photometry}

The ULP Cepheid sample and its mean, flux-weighted photometry in the standard system can be found in Table 1 . We assume that the photometric error associated with each ULP Cepheid is negligible when compared to the intrinsic scatter of the PL relation. We transform these measurements to absolute magnitudes via

$$
M_{i}=m_{i}-\mathrm{DM}-A_{i}, \quad i=I, V,
$$


Table 1

Ultra Long Period Cepheids

\begin{tabular}{|c|c|c|c|c|c|c|c|c|c|c|c|c|c|c|}
\hline $\begin{array}{l}\text { ID } \\
\text { (1) }\end{array}$ & $\begin{array}{l}\text { Host Galaxy } \\
\text { (2) }\end{array}$ & $\begin{array}{c}\text { R.A. } \\
\text { (J2000.0) } \\
(3)\end{array}$ & $\begin{array}{c}\text { Decl. } \\
(\mathrm{J} 2000.0) \\
(4)\end{array}$ & $\begin{array}{c}P \\
\text { (days) } \\
(5) \\
\end{array}$ & $\begin{array}{c}\langle V\rangle \\
(\mathrm{mag}) \\
(6) \\
\end{array}$ & $\begin{array}{c}(V-I) \\
(\mathrm{mag}) \\
(7) \\
\end{array}$ & $\begin{array}{c}W_{I} \\
(\mathrm{mag}) \\
(8) \\
\end{array}$ & $\begin{array}{c}E(B-V) \\
(\mathrm{mag}) \\
(9)\end{array}$ & $\begin{array}{c}(m-M)_{0} \\
(\mathrm{mag}) \\
(10)\end{array}$ & $\begin{array}{c}V_{0} \\
(\mathrm{mag}) \\
(11) \\
\end{array}$ & $\begin{array}{c}(V-I)_{0} \\
(\mathrm{mag}) \\
(12)\end{array}$ & $\begin{array}{c}W_{I 0} \\
(\mathrm{mag}) \\
(13) \\
\end{array}$ & $\begin{array}{c}12+\log (\mathrm{O} / \mathrm{H}) \\
(\operatorname{dex}) \\
(14)\end{array}$ & $\begin{array}{l}\text { References } \\
\text { (15) }\end{array}$ \\
\hline LMC HV883 & LMC & $05: 00: 08.6$ & $-68: 27: 03$ & 133.6 & 12.12 & 1.09 & 9.34 & 0.14 & 18.50 & -6.83 & 0.91 & -9.16 & $8.39 \pm 0.12$ & $1,2,3$ \\
\hline LMC HV2447 & LMC & $05: 19: 31.4$ & $-68: 41: 12$ & 118.7 & 11.99 & 1.12 & 9.13 & 0.14 & 18.50 & -6.96 & 0.94 & -9.37 & $8.39 \pm 0.12$ & $1,2,3$ \\
\hline LMC HV2883 & LMC & $04: 56: 27.0$ & $-64: 41: 26$ & 109.2 & 12.41 & 1.07 & 9.68 & 0.14 & 18.50 & -6.54 & 0.89 & -8.82 & $8.39 \pm 0.12$ & $1,2,3$ \\
\hline LMC HV5497 & LMC & $04: 55: 40.0$ & $-66: 25: 39$ & 98.6 & 11.92 & 1.11 & 9.09 & 0.14 & 18.50 & -7.03 & 0.93 & -9.41 & $8.39 \pm 0.12$ & $1,2,3$ \\
\hline SMC HV1956 & SMC & $01: 04: 15.9$ & $-72: 45: 20$ & 210.4 & 12.28 & 0.83 & 9.95 & 0.09 & 18.93 & -6.94 & 0.71 & -8.98 & $7.98 \pm 0.10$ & $5,2,3 ; 4$ \\
\hline SMC HV821 & SMC & $00: 41: 43.5$ & $-73: 43: 24$ & 127.5 & 11.92 & 1.03 & 9.29 & 0.09 & 18.93 & -7.30 & 0.92 & -9.64 & $7.98 \pm 0.10$ & $1,6,3 ; 4$ \\
\hline SMC HV829 & SMC & $00: 50: 28.9$ & $-72: 45: 09$ & 84.4 & 11.97 & 0.91 & 9.65 & 0.09 & 18.93 & -7.25 & 0.80 & -9.28 & $7.98 \pm 0.10$ & $1,6,3 ; 4$ \\
\hline NGC 6822-1 & NGC 6822 & $19: 45: 02.0$ & $-14: 47: 33$ & 123.9 & 17.86 & 1.40 & 14.29 & 0.36 & 23.31 & -6.60 & 0.94 & -9.02 & $8.11 \pm 0.10$ & $7,8,9$ \\
\hline NGC 55-1 & NGC 55 & $00: 14: 13.0$ & $-39: 08: 42$ & 175.9 & 19.25 & 0.84 & 17.11 & 0.13 & 26.43 & -7.60 & 0.68 & -9.33 & $8.05 \pm 0.10$ & $10,11,12$ \\
\hline NGC 55-2 & NGC 55 & $00: 15: 12.0$ & $-39: 12: 18$ & 152.1 & 19.56 & 0.95 & 17.14 & 0.13 & 26.43 & -7.28 & 0.79 & -9.29 & $8.05 \pm 0.10$ & $10,11,12$ \\
\hline NGC 55-3 & NGC 55 & $00: 14: 36.6$ & $-39: 11: 09$ & 112.7 & 20.18 & 1.05 & 17.51 & 0.13 & 26.43 & -6.67 & 0.88 & -8.92 & $8.05 \pm 0.10$ & $10,11,12$ \\
\hline NGC 55-4 & NGC 55 & $00: 15: 14.3$ & $-39: 13: 17$ & 97.7 & 20.54 & 1.25 & 17.35 & 0.13 & 26.43 & -6.31 & 1.08 & -9.08 & $8.05 \pm 0.10$ & $10,11,12$ \\
\hline NGC 55-5 & NGC 55 & $00: 15: 10.1$ & $-39: 12: 26$ & 85.1 & 20.84 & 1.38 & 17.32 & 0.13 & 26.43 & -6.01 & 1.22 & -9.12 & $8.05 \pm 0.10$ & $10,11,12$ \\
\hline NGC 300-1 & NGC 300 & $00: 55: 11.6$ & $-37: 33: 55$ & 115.8 & 20.13 & 0.97 & 17.66 & 0.10 & 26.37 & -6.55 & 0.85 & -8.71 & $8.25 \pm 0.22$ & $13,14,15$ \\
\hline NGC 300-2 & NGC 300 & $00: 54: 35.0$ & $-37: 35: 01$ & 89.1 & 19.71 & 1.02 & 17.12 & 0.10 & 26.37 & -6.97 & 0.89 & -9.25 & $8.25 \pm 0.22$ & $13,14,15$ \\
\hline NGC $300-3$ & NGC 300 & $00: 54: 54.3$ & $-37: 37: 02$ & 83.0 & 19.26 & 0.77 & 17.30 & 0.10 & 26.37 & -7.42 & 0.65 & -9.07 & $8.25 \pm 0.22$ & $13,14,15$ \\
\hline I Zw 18-1 & I Zw 18 & $\cdots$ & $\cdots$ & 129.8 & 23.56 & 0.74 & 21.67 & 0.03 & 31.30 & -7.84 & 0.70 & -9.63 & $7.21 \pm 0.10$ & 16,17 \\
\hline I Zw 18-2 & I Zw 18 & $\cdots$ & $\cdots$ & 125.0 & 23.47 & 0.91 & 21.15 & 0.03 & 31.30 & -7.93 & 0.87 & -10.15 & $7.21 \pm 0.10$ & 16,17 \\
\hline
\end{tabular}

Notes. ULP Cepheids in our sample grouped by host galaxy. (1) Cepheid Identification. (2) Host galaxy name. (3,4) Right ascension and declination in J2000 coordinates. (5) Period in days. (6) Apparen mean $V$ magnitude of Cepheid. (7) Apparent $(V-I)$ color of Cepheid. (8) Apparent Wesenheit magnitude (definition in the text). (9) Reddening toward host galaxy. (10) Reddening-free distance modulus. (11) Absolute $V$ magnitude. (12) Absolute $(V-I)$ color. (13) Absolute Wesenheit magnitude. (14) Metallicity: $12+\log (\mathrm{O} / \mathrm{H})$. (15) First reference is for photometry, reddening, and distance modulus (except where noted in the text). Second reference refers to metallicity. If third reference is present, its reddening and distance modulus measurements supercede the first.

References. 1. Freedman et al. 1985; 2. Pagel et al. 1978; 3. Udalski et al. 1999; 4. Hilditch et al. 2005 and Keller \& Wood 2006; 5. Pojmanski 1997, ASAS survey; 6. Peimbert \& Torres-Peimbert 1976; 7. Pietrzyński et al. 2004; 8. Peimbert et al. 2005; 9. Gieren et al. 2006; 10. Pietrzyński et al. 2006; 11. Tüllmann et al. 2003; 12. Gieren et al. 2008; 13. Gieren et al. 2004; 14. Urbaneja et al. 2005; 15. Gieren et al. 2005; 16. Fiorentino et al. 2008; 17. Skillman \& Kennicutt 1993. 

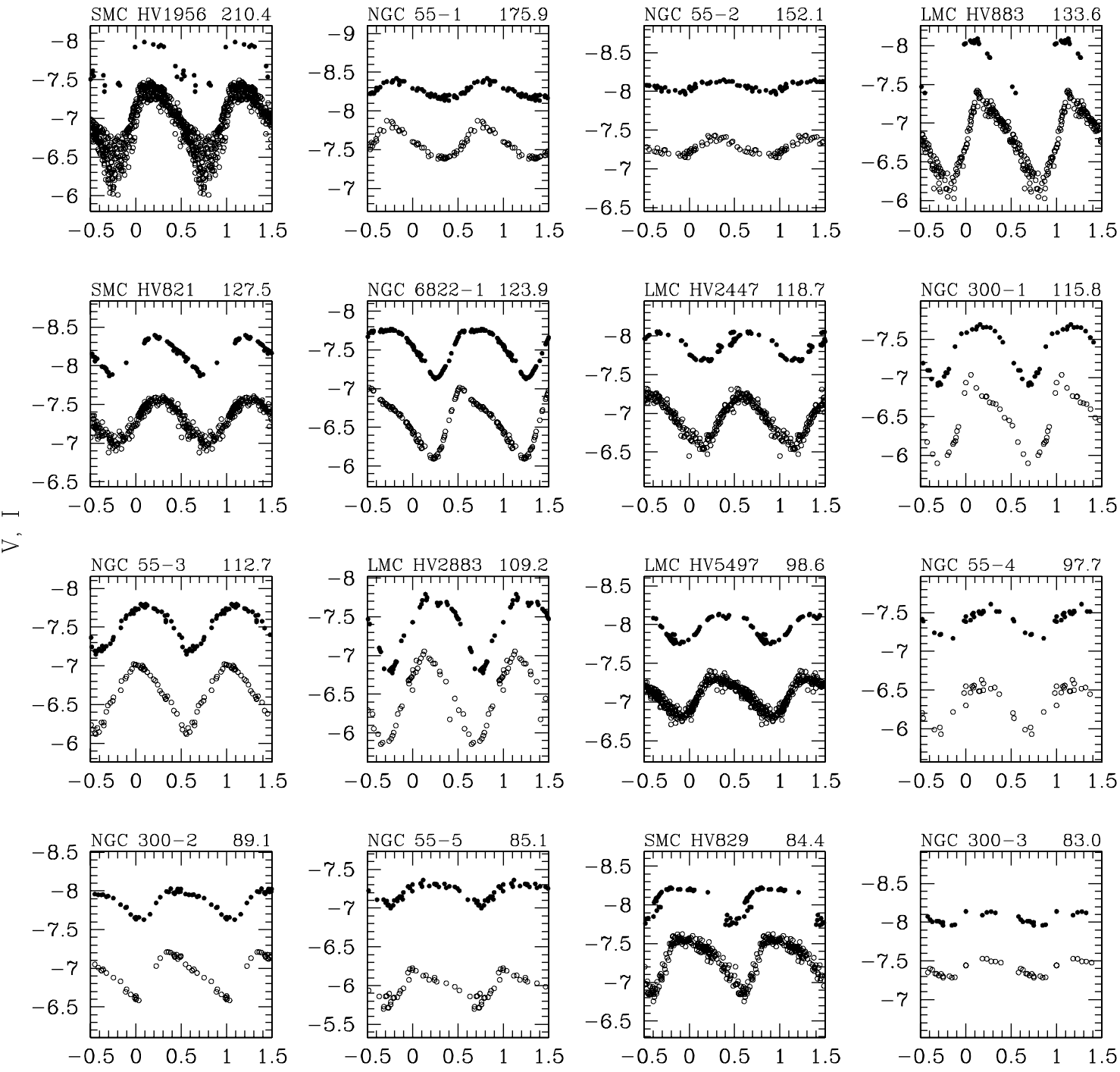

Phase

Figure 1. $V$ (open circles) and $I$ (filled circles, where available) band light curves of the ULP Cepheids. Each panel spans 2.4 mag along the $y$-axis. The phase is given along the $x$-axis. The Cepheid identification (see Column 1 of Table 1) is listed in the upper left of each plot while the period (in days) is in the upper right.

where $M_{i}$ is the absolute magnitude in either the $V$ or $I, m_{i}$ is the apparent magnitude; DM is the reddening free distance modulus; and $A_{i}$ is the extinction in the $V$ or $I$. We use the extinction law $A_{V}=3.24 E(B-V)$ and $A_{I}=1.96 E(B-V)$ (Schlegel et al. 1998). We define the Wesenheit magnitudes as $W_{I}=I-1.55(V-I)$ (e.g., Udalski et al. 1999).

The color-magnitude diagram (CMD) highlights several important characteristics of the ULP Cepheid data set (Figure 2). ULP Cepheids are the luminous counterparts to shorter period Cepheids in color-magnitude space. Our sample clearly populates the luminous region of the instability strip. Future Cepheid studies can use ULP Cepheids to push Cepheid distance measurements well beyond the current $\sim 30 \mathrm{Mpc}$ limit as our sample's median absolute magnitude is $M_{I}\left(M_{V}\right)=-7.86(-6.97)$ (see Section 5). The intrinsic brightness of ULP Cepheids makes them ideal candidates for distance indicators to galaxies where classical Cepheids cannot be observed.

\section{DISTANCE MEASUREMENTS WITH ULP CEPHEIDS}

The Cepheids in our sample have been ignored as distance indicators as they do not follow the standard PL relationship (e.g., Freedman et al. 1992). In this section, we examine the characteristics of our ULP Cepheid sample in the PL plane and explore their viability as a distance indicator. We determine PL relations of our sample in the $V, I$, and $W_{I}$ in Section 3.1 while the metallicity dependence of our results is presented in Section 3.2.

\subsection{Period-Luminosity Relations}

Using the data in Table 1, we construct PL diagrams in $V, I$, and $W_{I}$ (Figures 3-5, respectively). In each case, ULP Cepheids are compared to fundamental mode SMC Cepheids (hereafter, this control sample will be referred to as OGLE Cepheids). The OGLE Cepheid sample contains over 1100 fundamental mode Cepheids with periods ranging from 0.5 to $\sim 50$ days; however, we plot only the 70 Cepheids with periods greater than 10 days. To quantify our comparison, we perform a linear fit on both samples in each PL diagram: the slopes of the OGLE Cepheid PL relations (hereafter PL $_{S M C}$; the dotted lines in the figures) are adopted from Udalski et al. (1999), while the intercepts are chosen to minimize $\chi^{2}$. We employ linear least-square fitting of the ULP Cepheid sample to identify their PL relation (hereafter 


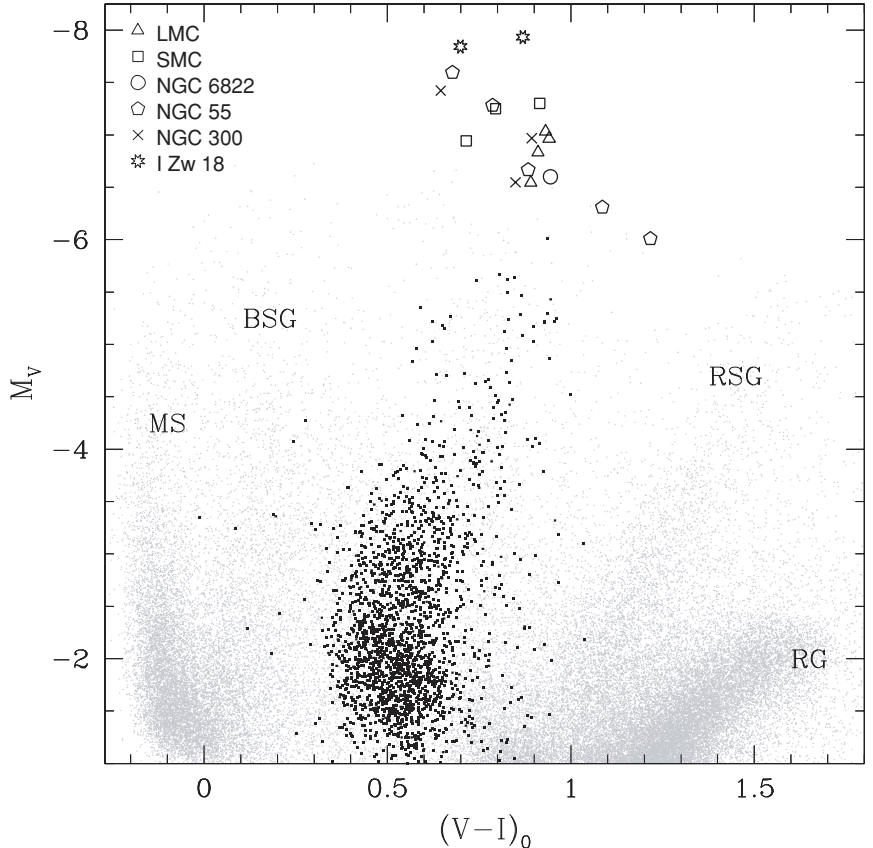

Figure 2. $M_{V}$ vs. $(V-I)_{0}$ color CMD of our ULP sample (open symbols) with the OGLE SMC Cepheids (black dots) and OGLE SMC stars (gray dots) for comparison. The legend denotes the host galaxy of each ULP Cepheid For reference we label the main sequence (MS), blue supergiant (BSG), red supergiant (RSG), and red giant (RG) sequences.

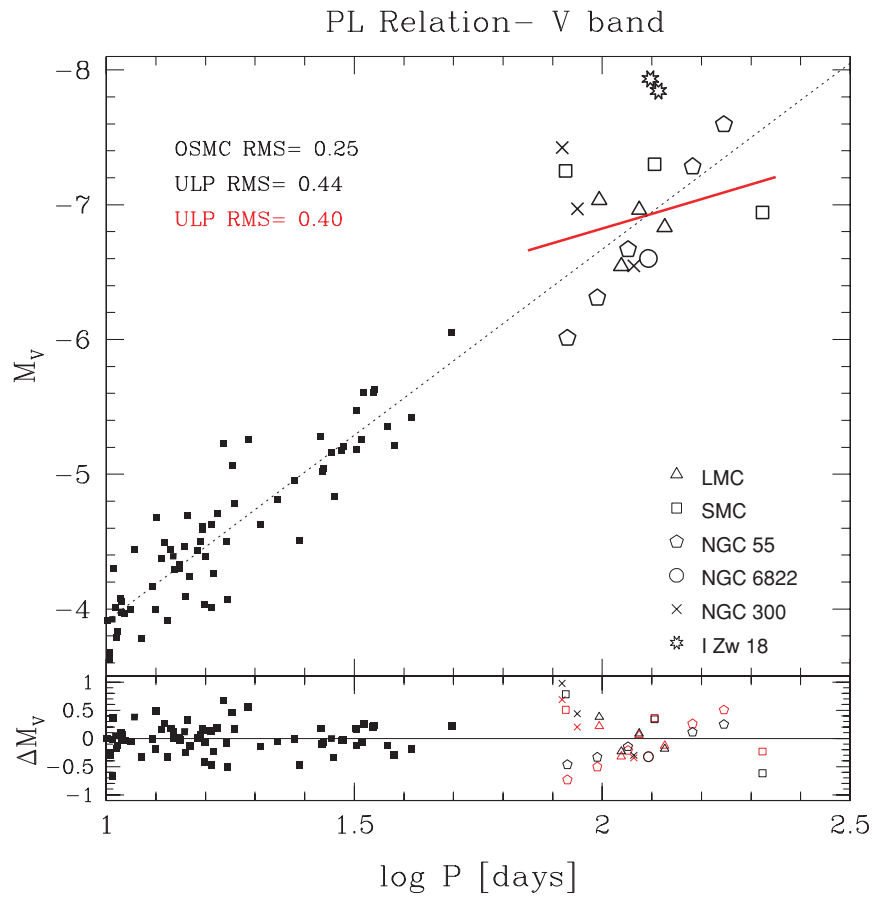

Figure 3. $V$ PL relationship for the OGLE SMC Cepheids (black dots) and ULP Cepheids (open symbols). The dashed line is the PL relation adopted from Udalski et al. (1999), with a slope of -2.76 . The least-square fit of the ULP Cepheid subsample yields a flatter slope of $-1.09 \pm 0.94$ (red line) and the rms is $0.40 \mathrm{mag}$. The residuals to the PLSMC fit are shown in the bottom panel (black, open squares). Residuals to the $\mathrm{PL}_{\mathrm{ULP}}$ are given for the ULP Cepheid sample (red symbols).

(A color version of this figure is available in the online journal.)

$\left.\mathrm{PL}_{\mathrm{ULP}}\right)$. We omit errors in distance moduli and extinction in this demonstration as they are small when compared to the overall scatter of the sample. The parameters of these fits and the rms

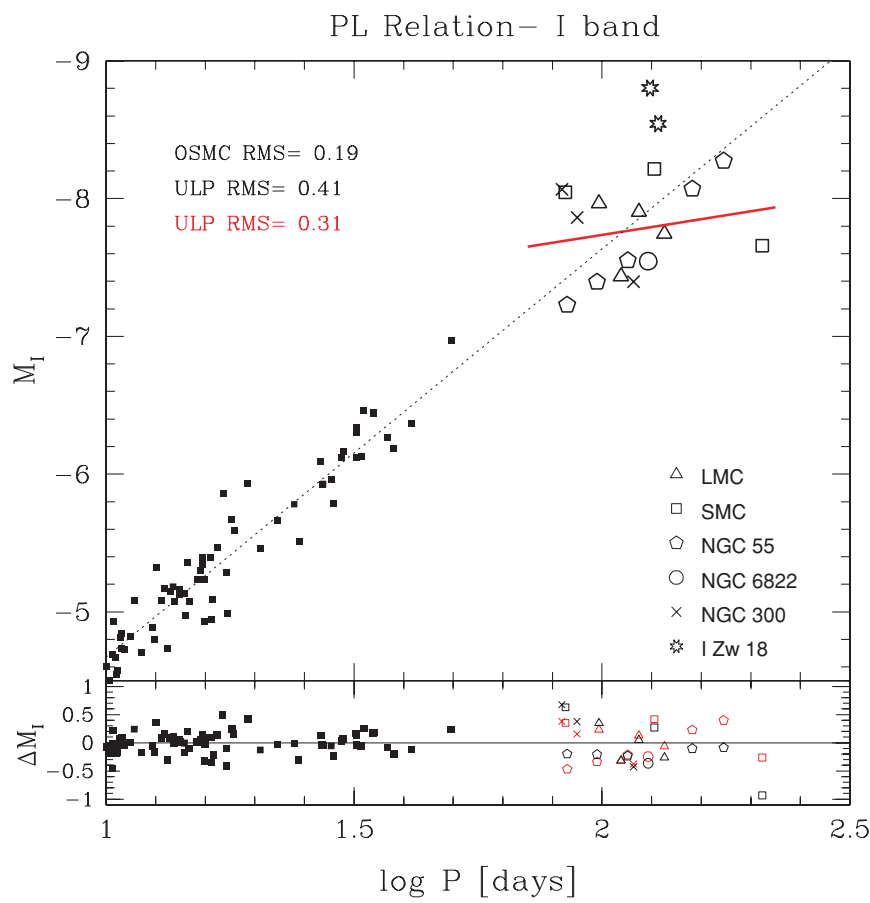

Figure 4. I PL relationship for the OGLE SMC Cepheids (black dots) and ULP Cepheids (open symbols). The dashed line has a slope of -2.96 and is the PL relation adopted from Udalski et al. (1999). The least-square fit to the ULP Cepheid subsample produces a flatter slope of $-0.57 \pm 0.73$ (red line) and the rms is $0.31 \mathrm{mag}$. The residuals to the PLSMC fit are shown in the bottom panel (black, open squares). Residuals to the PLULP are given for the ULP Cepheid sample (red symbols).

(A color version of this figure is available in the online journal.)

of each data set in $V, I$, and $W_{I}$ are listed in Table 2. Despite our sample ranging in period from 83 to 210 days, ULP Cepheids occupy a small region of luminosity space. We note that the ULP Cepheids from I Zw 18 are not included in this analysis for reasons outlined in Section 2.

The $V$ PL diagram is shown in Figure 3 . The $\mathrm{PL}_{\mathrm{SMC}}$ fit has a slope of -2.76 and the rms of the OGLE Cepheid sample about this fit is 0.25 mag. The ULP Cepheid sample has $76 \%$ more scatter (rms $=0.44 \mathrm{mag}$ ) about the $\mathrm{PL}_{\mathrm{SMC}}$ fit. This discrepancy in scatter has led to the standard practice of removing ULP Cepheids from PL relation studies (e.g., Freedman et al. 1985) and the significant increase in rms suggests that the ULP Cepheids do not conform to the classical PL relation. If we determine the PL relation of ULP Cepheids alone we find PL $\mathrm{ULP}_{\mathrm{U}}$ has a slope $(-1.09 \pm 0.94)$ that is flatter than PL $_{S M C}$ (though the slopes are within $2 \sigma$ of each other, see Table 2). The rms of our sample to $\mathrm{PL}_{\mathrm{ULP}}$ is $0.40 \mathrm{mag}$; only marginally better than the ULP Cepheid scatter about the established PL SMC $_{\text {relation. }}$ In $V$, the ULP Cepheid sample does not follow a statistically distinct and unique PL.

The longer the wavelength the less reddening is a concern. The accuracy of distance measurement with Cepheids increases moving from $V$ to $I$ (the PL diagram in Figure 4). PL $_{S M C}$ has a slope of -2.96 and the OGLE Cepheid sample's rms is 0.19 . The ULP Cepheid scatter about this fit is $116 \%$ larger (0.41 mag). The rms of the ULP Cepheids is reduced to 0.31 mag when using the $\mathrm{PL}_{\mathrm{ULP}}$ fit (slope $=-0.57 \pm 0.73$ ). $\mathrm{PL}_{\mathrm{ULP}}$ is approximately five times as flat as $\mathrm{PL}_{\mathrm{SMC}}$ and the two slopes are distinct at the $3 \sigma$ level. While ULP Cepheids show the same general trends with regards to period, luminosity, and color as normal Cepheids, significant statistical differences 
Table 2

Least-Square Fit Values: $y=b+a \times x$

\begin{tabular}{|c|c|c|c|c|c|}
\hline $\begin{array}{l}\text { Relation } \\
\text { (1) }\end{array}$ & $\begin{array}{l}\text { Subset } \\
(2)\end{array}$ & $\begin{array}{c}\text { Shorthand } \\
\text { (3) }\end{array}$ & $\begin{array}{c}\text { Intercept (b) } \\
\text { (4) }\end{array}$ & $\begin{array}{c}\text { Slope (a) } \\
\text { (5) }\end{array}$ & $\begin{array}{c}\mathrm{rms} \\
(6)\end{array}$ \\
\hline Period-luminosity: $V$ & SMC Cepheids & $\mathrm{PL}_{\mathrm{SMC}, V}$ & -1.15 & -2.76 & 0.25 \\
\hline Period-luminosity: $V$ & ULPs & PLULP, $V$ & $-4.64 \pm 1.95$ & $-1.09 \pm 0.94$ & 0.40 \\
\hline Period-luminosity: I & SMC Cepheids & $\mathrm{PL}_{\mathrm{SMC}, I}$ & -1.71 & -2.96 & 0.19 \\
\hline Period-luminosity: $I$ & ULPs & $\mathrm{PL}_{\mathrm{ULP}, I}$ & $-6.58 \pm 1.50$ & $-0.57 \pm 0.73$ & 0.31 \\
\hline Period-luminosity: $W_{I}$ index & SMC Cepheids & $\mathrm{PL}_{\mathrm{SMC}, W_{I}}$ & -2.57 & -3.28 & 0.12 \\
\hline Period-luminosity: $W_{I}$ index & ULPs & $\mathrm{PL}_{\mathrm{ULP}, W_{I}}$ & $-9.06 \pm 1.12$ & $-0.05 \pm 0.54$ & 0.23 \\
\hline
\end{tabular}

Notes. The fit values of the PL relationships in $V, I$, and $W_{I}$. For each photometric system, we calculate the PL relation of classical SMC (PL $\mathrm{SMC}_{\text {) }}$ and ULP (PLULP) Cepheids.

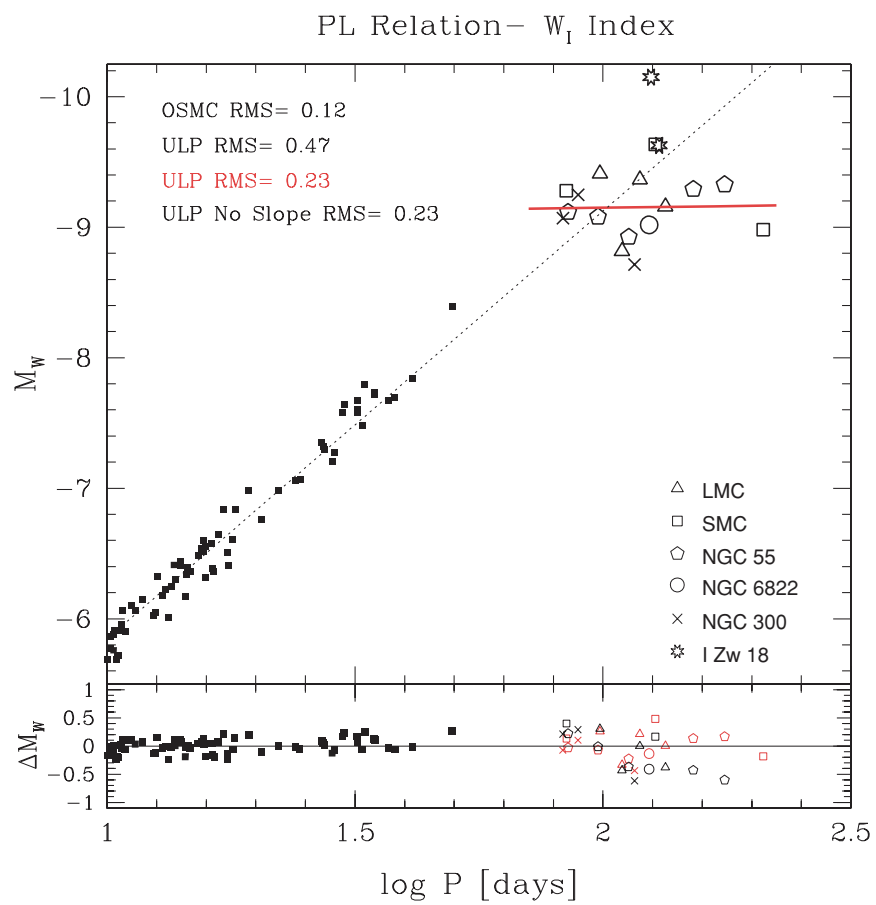

Figure 5. Absolute Wesenheit magnitude PL relationship for the OGLE SMC Cepheids (black dots) and ULP Cepheids (open symbols). The dashed line is the PL relation adopted from Udalski et al. (1999) and has a slope of -3.28. The least-square fit to the ULP Cepheid subsample produces a flat slope of $-0.05 \pm 0.54$ (red line) with a ULP Cepheid rms $=0.23 \mathrm{mag}$. If we assume that $\mathrm{PL}_{\mathrm{ULP}}$ has zero slope (intercept of -9.15 ), the rms stays at $0.23 \mathrm{mag}$. The residuals to the PLSMC fit are shown in the bottom panel (black, open squares). Residuals to the PL $L_{U L P}$ are given for the ULP Cepheid sample (red symbols).

(A color version of this figure is available in the online journal.)

between the two populations are apparent in the I-band PL relation.

To further reduce the uncertainty associated with reddening in our PL analysis, we repeat the procedure using the reddeningfree Wesenheit Index $\left(W_{I}\right)$ introduced in Madore \& Freedman (1991). The $W_{I}$ PL diagram illuminates the advantages of this reddening-free index for Cepheid distance measurements (Figure 5). The OGLE Cepheid sample has a very tight relation between period and luminosity; with small scatter about the $\mathrm{PL}_{\mathrm{SMC}}$ fit (slope of -3.28 ; rms of only $0.12 \mathrm{mag}$ ). The ULP Cepheid rms about $\mathrm{PL}_{\mathrm{SMC}}$ is $0.47 \mathrm{mag}$. The fourfold increase in scatter implies that the ULP Cepheid and the OGLE Cepheid samples do not conform to the same PL relation. The PL $\mathrm{ULP}_{\mathrm{LP}}$ fit slope is flatter than its $\mathrm{PL}_{\mathrm{SMC}}$ counterpart at the $6 \sigma$ level $(-0.05 \pm 0.54$ versus -3.28$)$ and the ULP fit slope is consistent with zero slope. The scatter of the ULP Cepheids is only
0.23 mag about the $\mathrm{PL}_{U L P}$ relation. This scatter is still $92 \%$ more than that of the OGLE Cepheid sample; however, the ULP sample is relatively small and heterogeneous. We note that the scatter of ULP Cepheids about the PL ULP fit is smaller than that of the OGLE Cepheid sample about the nominal PL relation in $V$ and on par with the same in $I$.

Several trends in PL space are apparent as one examines the ULP Cepheid sample in $V$, then $I$, and finally in the $W_{I}$ index. As reddening is reduced, the $\mathrm{PL}_{\mathrm{ULP}}$ parameters are increasingly different from those of $\mathrm{PL}_{\mathrm{SMC}}$. The $\mathrm{PL}_{\mathrm{ULP}}$ fit is more shallow as one moves from $V$ to $W_{I}$. In the $W_{I}$, the PL $\mathrm{PLP}_{\mathrm{ULP}}$ relation reveals that ULP Cepheid luminosity becomes statistically independent of period. In essence, ULP Cepheids behave as bright, standard candles in the reddening-free index.

\subsection{Metallicity Dependence}

An uncertainty of the Cepheid PL and its derived distance measurement is its sensitivity to the metallicity of the stars (e.g., Freedman \& Madore 1990; Kennicutt et al. 1998). Our sample of ULP Cepheids contains six different host galaxies that span a range of $\sim 1.2$ dex in $12+\log (\mathrm{O} / \mathrm{H})$ from 7.22 to 8.39 , providing an opportunity to investigate the dependence of the ULP Cepheid PL on metallicity. We plot the residual of each ULP Cepheid to the PLULP, $W_{I}$ fit listed in Table 2 as a function of metallicity (Figure 6). In other studies, linear fits of PL residuals have determined a correction factor, $\gamma$, between 0.0 and $-0.4 \mathrm{mag} \mathrm{dex}^{-1}$ (see Figure 1 of Romaniello et al. 2008). Recently, Macri et al. (2006) used the metallicity gradient in NGC 4258 to determine $\gamma=-0.29 \pm 0.09 \pm 0.05$ mag dex $^{-1}$ (random and systematic errors, respectively). We overlay this relation (normalized to our data set) in Figure 6 for reference. If the I $\mathrm{Zw} 18$ Cepheids are confirmed, it suggests a stronger correlation between PL offset and metallicity than is evident in lower period Cepheids (e.g., Kochanek 1997; Kennicutt et al. 1998). However, we note that we do not take into account any reddening or DM errors in this analysis. As such, we do not claim a specific metallicity dependence for ULP Cepheids. We simply demonstrate that the residuals to the PL $\mathrm{ULP}_{\mathrm{L}}$ fit are broadly consistent with the range of values presented in the literature to date.

At this time we do not apply a metallicity correction to our ULP Cepheid PL relations. Precise gas-phase oxygen abundance measurements are difficult to obtain (for a review see Bresolin 2006) and we adopt a minimum metallicity error of 0.1 dex. The ULP Cepheid sample must grow in size and the PL analysis must be more detailed to determine if I $\mathrm{Zw} 18$ is an exception and to characterize the functional form of the ULP Cepheid PL sensitivity to metallicity. 


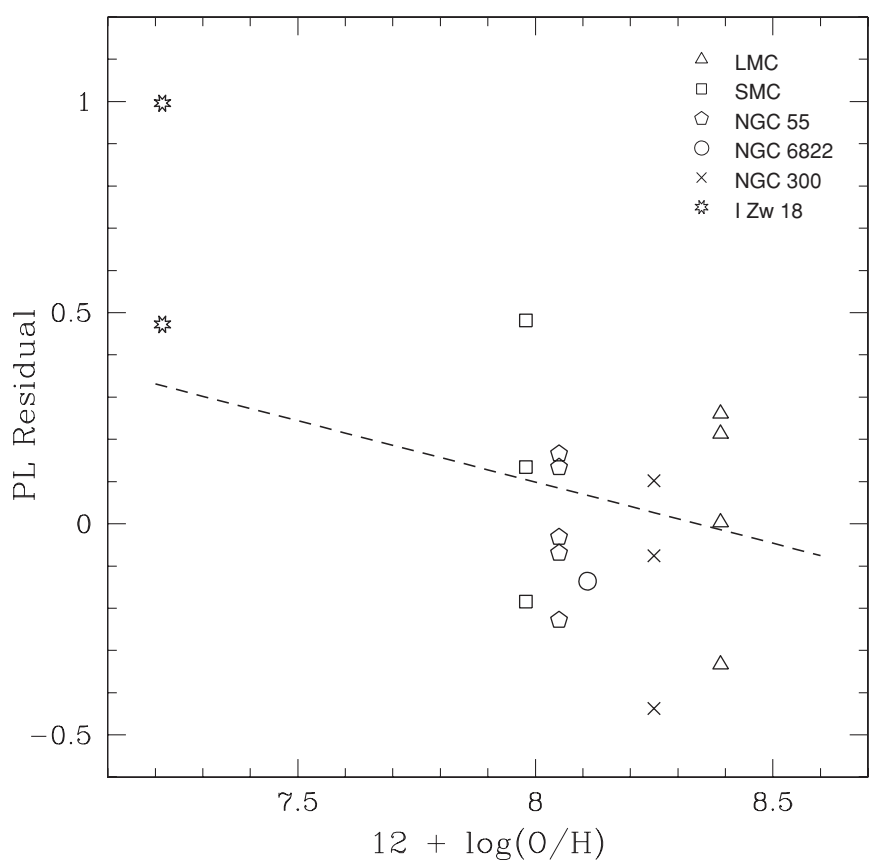

Figure 6. Residual to the Wesenheit index PL relation (see Table 2, PL $\mathrm{ULP}_{W_{I}}$ ) vs. metallicity. The open symbols are ULP Cepheids from the sample. The dotted line corresponds to the luminosity correction of $-0.29 \pm 0.10 \mathrm{mag} \mathrm{dex}^{-1}$ determined by Macri et al. (2006) and normalized to our sample. The residuals of the sample, minus the two I Zw 18 Cepheids, are consistent with the Macri et al. (2006) result.

\section{USING ULP CEPHEIDS TO PROBE THE EVOLUTIONARY MODELS OF MASSIVE STARS}

Most Cepheid variables cross the instability strip three times (e.g., Bono et al. 2000; Pietrukowicz 2001). One can determine which crossing a Cepheid is undergoing by measuring its period change, $d P / d t$. The first and third crossings are associated with positive $d P / d t$, while Cepheids exhibit a decreasing period on their second crossing. We investigated the light curves of our sample for signs of period changes by comparing photometry taken over the last 30 years. One ULP Cepheid, HV829, exhibits a negative period change of about 1.5 days. For this Cepheid, we compiled photometric data taken during 1970-1976 from Madore (1975) and van Genderen (1983) and 2000-2004 data from the ASAS catalog (see Figure 7). This result is confirmed by a measured period of 87.63 days in Payne-Gaposchkin \& Gaposchkin (1966) and 85.2 days by Moffett et al. (1998), firmly establishing HV829 as a Cepheid on its second crossing. No other Cepheid in our sample exhibited a measurable period change.

ULP Cepheids occupy a mass range that is ideal to probe high-mass evolutionary models along the instability strip. We plot the CMD of our ULP Cepheid sample and overlay the evolutionary tracks of Lejeune \& Schaerer (2001; see Figure 8). The location of ULP Cepheids in the CMD suggests masses between 13 and $20 M_{\odot}$, depending on the assumed metallicity (here, we choose SMC metallicity). Our sample clearly probes a mass range unexplored by current Cepheid studies. Note that the evolutionary tracks at 15 and $20 M_{\odot}$ only cross the instability strip once, regardless of assumed mass-loss rate. However, we have shown and confirmed that HV829 is undergoing its second crossing; a result at odds with the models. We note that the evolutionary model represents some "mean" behavior based on assumptions of stellar chemical composition, overshooting, and other parameters. Nevertheless, our result is one of the few observational constraints on high-mass stellar evolutionary models. HV829 may indicate that massive stars behave differently than expected. Future stellar evolutionary models in this mass regime should take this into account.

\section{DISCUSSION}

We have presented, for the first time, a collection of ULP ( $P>80$ days) Cepheids from the literature and demonstrated their viability as a distance indicator. In the past, ULP Cepheids have been ignored as distance indicators, and in fact many stellar variability searches did not extend their cadence and search strategy to allow for their discovery.

In $V, I$, and especially reddening-free $W_{I}$ magnitude, ULP Cepheids have a relatively flat PL relation compared to the respective relations for classical Cepheids (Udalski et al. 1999). The dispersion in both the classical and ULP Cepheid populations about their respective PL relationships becomes smaller as one moves from $V$ to $I$ to $W_{I}$, and the discrepancy between the slopes increases. Most notably, the slope of the ULP Cepheid $W_{I}$ PL relation is significantly shallower than the standard SMC PL relation (slope is -0.05 versus -3.28 ). The reddening-free Wesenheit index produces the tightest ULP PL relation, with rms residual of only 0.23 mag (see Figure 5). Other papers (e.g., Kanbur et al. 2007) have found nonlinearities in the PL relationship at lower periods. However, the change in slope seen here is much more dramatic and it is unlikely that it shares a physical connection with the PL changes at lower periods.

Our ULP PL scatter in $W_{I}$ is already less than that of the initial peak brightness versus absolute magnitude relation for Type Ia Supernova ( $0.3 \mathrm{mag}$; Phillips 1993). A huge amount of effort has been necessary to increase the number of observed Type Ia $\mathrm{SNe}$, refine the calibration technique (e.g., Prieto et al. 2006), and to obtain the low $0.15-0.20$ mag scatter in the relation seen today (e.g., Jha et al. 2007). We expect that future observational and theoretical studies of ULP Cepheids will further decrease the already fairly small scatter found in this first analysis.

We strove to find all the known ULP Cepheids in the literature, but our sample is likely not a complete census of ULP Cepheids. We note that the Araucaria Project found ULP Cepheids in three of the five galaxies they observed at the time of this analysis, so the ULP Cepheid sample should grow at a reasonable rate as Cepheid studies are extended to more galaxies and previous data sets are reanalyzed with longer period searches. There is also evidence that ULP Cepheids exist in a broad range of metallicities, as preliminary data analysis of a M81 variability survey with the Large Binocular Telescope has already discovered at least one ULP Cepheid (C. S. Kochanek 2009, private communication).

In addition to following a fairly tight PL relation, the ULP Cepheids are also very luminous, with a median absolute magnitude in $I(V)$ for our sample of $-7.86(-6.97)$. Using WFPC3, $H S T$ can obtain $10 \%$ photometry at $V=28$ or $I=27$ (DM $=35$-distance of $100 \mathrm{Mpc}$ for the median ULP Cepheid) in about 10 orbits, while only two orbits are needed to reach a DM of 34. As one would only need a few orbits per epoch, one could detect the median ULP Cepheid (with a period of $\sim 121$ days) at $100 \mathrm{Mpc}$ and the brightest ULP Cepheids at $\sim 150 \mathrm{Mpc}$ in a reasonable amount of time. Since the luminosity of ULP Cepheid is a weak function of its period, relatively accurate distances would not require as precise period measurements as is needed for classical Cepheids. We encourage future 


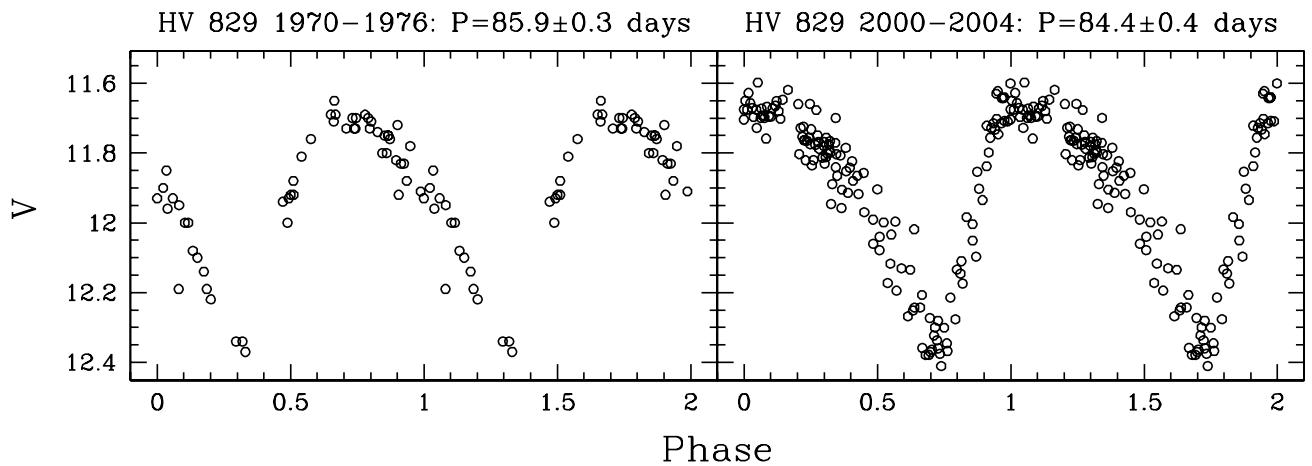

Figure 7. $V$-band photometry of SMC HV829 taken from 1970 to 1976 (left panel) and 2000 to 2004 (right panel; see the text for data references). Each set of data was phased with the period shown at the top of each panel. The pulsation period has clearly decreased, from 85.9 days to 84.4 days over approximately 30 years.

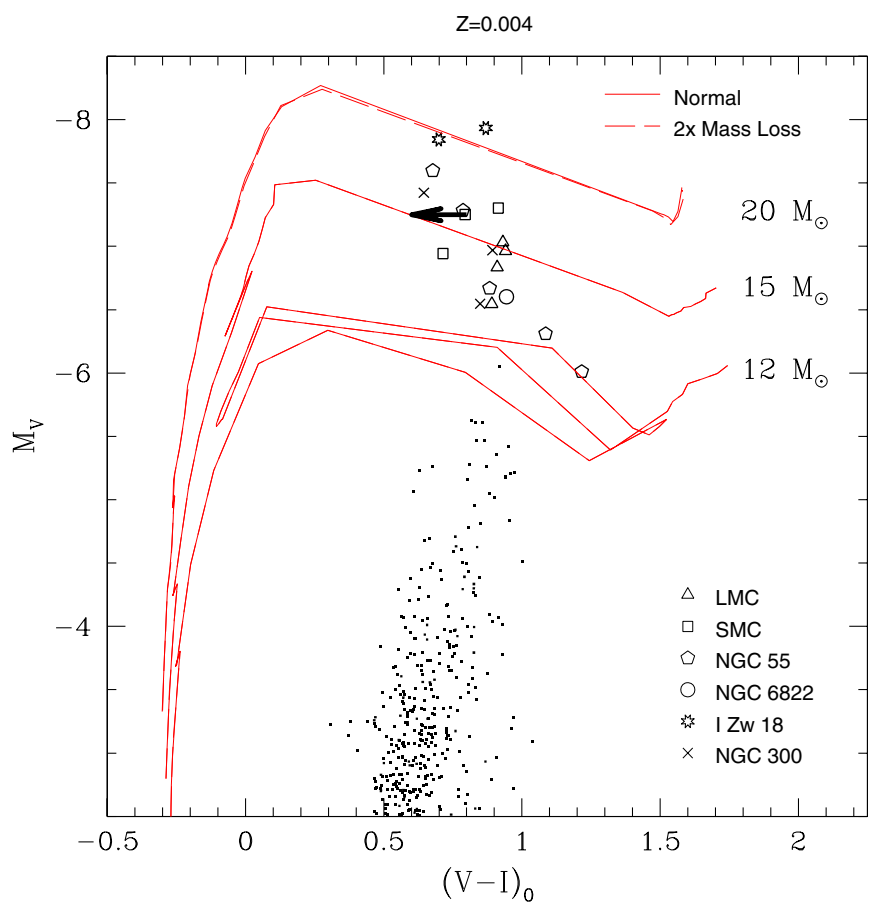

Figure 8. CMD of OGLE SMC Cepheids (small blacks dots) and ULP Cepheids (large open symbols) overlaid with $12 M_{\odot}, 15 M_{\odot}$, and $20 M_{\odot}$ evolutionary models of Lejeune \& Schaerer (2001). The metallicity chosen for the models is similar to the SMC. For each mass, models incorporating "normal" (solid line) mass loss and "double" (dashed line) mass loss are plotted. The two mass-loss models produce essentially the same evolutionary track for each mass plotted. The large arrow denotes that the pulsation period of HV829 is becoming smaller; therefore, the star is undergoing its second crossing of the instability strip.

(A color version of this figure is available in the online journal.)

variability proposals to search for Cepheids with periods greater than 100 days.

We note two concerns in using ULP Cepheids for distance measurements. It is obvious from our sample size that ULP Cepheids are far less common than classical Cepheids. The relatively small ULP Cepheid population will make precision distance measurements less practical. As the sample size grows and the ULP Cepheid PL relations become established, a single ULP Cepheid observation may yield distance measurements accurate to $10 \%-20 \%$. Blending is always a concern in Cepheid studies. Blending can compromise classical Cepheid measurements as stars of comparable brightness are likely to lie within a single PSF (Mochejska et al. 2000), especially at larger distances. We expect blending to be less of a problem for our ULP Cepheids even at large distances simply because they are so bright and there are very few stars of comparable brightness in a given galaxy. The effect of blending on ULP Cepheid observations will need to be investigated further as the sample is increased.

The data set and analysis herein provides meaningful constraints on theoretical work on Cepheids in this mass and period range. We have shown that ULP Cepheids show strong evidence for a different, flatter PL relation than their lower period cousins. Several papers have modeled Cepheid pulsations and mapped these to theoretical PL relations (e.g., Bono et al. 2002). However, this work has not been reliably extended to the period range of our sample. Pulsation models in this period range would also help determine the intrinsic scatter to the $\mathrm{PL}_{\mathrm{ULP}}$ relation.

Our current sample is not large enough to constrain the sensitivity of the ULP Cepheid PL to metallicity. The PL residuals as a function of metallicity are consistent with the results for shorter period Cepheids $(\gamma=-0.29 \pm 0.09 \pm 0.05$ mag $\mathrm{dex}^{-1}$; Macri et al. 2006). Note that the median $12+$ $\log (\mathrm{O} / \mathrm{H})$ value for a galaxy in our sample is about 0.5 dex lower than the corresponding value for the HST Key Project Cepheid hosts.

Period changes of ULP Cepheids have powerful implications for stellar evolutionary models in this mass regime. We examined photometry from two epochs separated by 30 years to check for evidence of period changes in seven Magellanic Cloud ULP Cepheids. Only one, SMC HV829, showed a significant period change - from $85.9 \pm 0.3$ to $84.4 \pm 0.4$ days. The negative period derivative indicates that the Cepheid is undergoing its second crossing. Assuming the metallicity of the SMC, many current evolutionary models do not predict second crossings for Cepheids in this mass range (see Figure 8). Future models should incorporate this observational result as it should place limits on several key input parameters. In addition to their potential as distance indicators, ULP Cepheids provide observational constraints on high-mass stellar evolution models.

To summarize, ULP Cepheids, while often dismissed in the past, are potentially a powerful distance indicator, probe of PL metallicity sensitivity, and also a probe of massive star evolutionary models. ULP Cepheids could provide the first direct stellar distance measurements to galaxies in the 50 $150 \mathrm{Mpc}$ range, extending the cosmological distance ladder well into the Hubble flow.

We would like to thank Chris Kochanek for his thoughtful comments on the manuscript. We thank the referee for improving this work with helpful comments. This work was supported by NSF grant AST-0707982. 


\section{REFERENCES}

Aloisi, A., et al. 2007, ApJ, 667, L151

Baade, W. 1956, PASP, 68, 5

Bessel, F. W. 1839, Astron. Nachr., 16, 65

Bono, G., Caputo, F., Cassisi, S., Marconi, M., Piersanti, L., \& Tornambè, A. 2000, ApJ, 543, 955

Bono, G., Groenewegen, M. A. T., Marconi, M., \& Caputo, F. 2002, ApJ, 574, L33

Bresolin, F. 2006, arXiv:astro-ph/0608410

Chiosi, C., Wood, P. R., \& Capitanio, N. 1993, ApJS, 86, 541

Fiorentino, G., et al. 2008, Mem. Soc. Astron. Ital., 79, 461

Freedman, W. L., Grieve, G. R., \& Madore, B. F. 1985, ApJS, 59, 311

Freedman, W. L., \& Madore, B. F. 1990, ApJ, 365, 186

Freedman, W. L., Madore, B. F., Hawley, S. L., Horowitz, I. K., Mould, J., Navarrete, M., \& Sallmen, S. 1992, ApJ, 396, 80

Freedman, W. L., et al. 2001, ApJ, 553, 47

Gieren, W., Pietrzyński, G., Nalewajko, K., Soszyński, I., Bresolin, F., Kudritzki, R.-P., Minniti, D., \& Romanowsky, A. 2006, ApJ, 647, 1056

Gieren, W., Pietrzyński, G., Soszyński, I., Bresolin, F., Kudritzki, R.-P., Minniti, D., \& Storm, J. 2005, ApJ, 628, 695

Gieren, W., Pietrzyński, G., Soszyński, I., Bresolin, F., Kudritzki, R.-P., Storm, J., \& Minniti, D. 2008, ApJ, 672, 266

Gieren, W., et al. 2004, AJ, 128, 1167

Grieve, G. R., Madore, B. F., \& Welch, D. L. 1985, ApJ, 294, 513

Hilditch, R. W., Howarth, I. D., \& Harries, T. J. 2005, MNRAS, 357, 304

Jha, S., Riess, A. G., \& Kirshner, R. P. 2007, ApJ, 659, 122

Kanbur, S. M., Ngeow, C., Nanthakumar, A., \& Stevens, R. 2007, PASP, 119, 512

Keller, S. C., \& Wood, P. R. 2006, ApJ, 642, 834

Kennicutt, R. C. Jr., et al. 1998, ApJ, 498, 181

Kochanek, C. S. 1997, ApJ, 491, 13

Leavitt, H. S. 1908, Ann. Harvard College Obs., 60, 87

Lejeune, T., \& Schaerer, D. 2001, A\&A, 366, 538

Macri, L. M. 2005, arXiv:astro-ph/0507648
Macri, L. M., Stanek, K. Z., Bersier, D., Greenhill, L. J., \& Reid, M. J. 2006, ApJ, 652,1133

Madore, B. F. 1975, ApJS, 29, 219

Madore, B. F., \& Freedman, W. L. 1991, PASP, 103, 933

Mochejska, B. J., Macri, L. M., Sasselov, D. D., \& Stanek, K. Z. 2000, AJ, 120 , 810

Moffett, T. J., Gieren, W. P., Barnes, T. G. III, \& Gomez, M. 1998, ApJS, 117, 135

Pagel, B. E. J., Edmunds, M. G., Fosbury, R. A. E., \& Webster, B. L. 1978, MNRAS, 184,569

Payne-Gaposchkin, C., \& Gaposchkin, S. 1966, Smithsonian Contrib. Astrophys., 9, 1

Peimbert, A., Peimbert, M., \& Ruiz, M. T. 2005, ApJ, 634, 1056

Peimbert, M., \& Torres-Peimbert, S. 1976, ApJ, 203, 581

Phillips, M. M. 1993, ApJ, 413, L105

Pietrukowicz, P. 2001, Acta Astron., 51, 247

Pietrzyński, G., Gieren, W., Fouqué, P., \& Pont, F. 2002, AJ, 123, 789

Pietrzyński, G., Gieren, W., Udalski, A., Bresolin, F., Kudritzki, R.-P., Soszyński, I., Szymański, M., \& Kubiak, M. 2004, AJ, 128, 2815

Pietrzyński, G., et al. 2006, AJ, 132, 2556

Pojmanski, G. 1997, Acta Astron., 47, 467

Prieto, J. L., Rest, A., \& Suntzeff, N. B. 2006, ApJ, 647, 501

Riess, A. G., et al. 2004, ApJ, 607, 665

Romaniello, M., et al. 2008, A\&A, 488, 731

Sandage, A., Tammann, G. A., \& Reindl, B. 2008, arXiv:0810.1780v1

Schlegel, D. J., Finkbeiner, D. P., \& Davis, M. 1998, ApJ, 500, 525

Schwarzenberg-Czerny, A. 1989, MNRAS, 241, 153

Skillman, E. D., \& Kennicutt, R. C., Jr. 1993, ApJ, 411, 655

Spergel, D. N., et al. 2003, ApJS, 148, 175

Stanek, K. Z., \& Udalski, A. 1999, arXiv:astro-ph/9909346

Tegmark, M., et al. 2004, Phys. Rev. D, 69, 103501

Tüllmann, R., Rosa, M. R., Elwert, T., Bomans, D. J., Ferguson, A. M. N., \& Dettmar, R.-J. 2003, A\&A, 412, 69

Udalski, A., Szymanski, M., Kubiak, M., Pietrzynski, G., Soszynski, I., Wozniak, P., \& Zebrun, K. 1999, Acta Astron., 49, 201

Urbaneja, M. A., et al. 2005, ApJ, 622, 862

van Genderen, A. M. 1983, A\&AS, 52, 423 